\title{
VOXEL-MAN TempoSurg
}

\section{A Virtual Reality Temporal Bone Surgery Simulator}

\author{
RUDOLF LEUWER $^{1)}$, ANDREAS PETERSIK ${ }^{2)}$, BERNHARD PFLESSER ${ }^{2)}$, ANDREAS \\ POMMERT $^{2)}$, BORIS TOLSDORFF ${ }^{3)}$, KARL-HEINZ HÖHNE ${ }^{2)}$, ULF TIEDE ${ }^{2)}$ \\ 1) University of Düsseldorf Medical School at Krefeld, Helios Hospital \\ 2) Voxel-Man Group, University Medical Center Hamburg-Eppendorf, Hamburg, Germany \\ 3) ENT Department, University Medical Center Hamburg-Eppendorf, Hamburg, Germany
}

Summary: Objectives: There is a growing demand for the development of virtual reality surgical simulators. Due to the complex surgical anatomy of the skull base and midface such a simulator has very special requirements concerning accuracy of visualization, cutting process and haptic force feedback.

Methods: The anatomical 3D-model of the VOXEL-MAN TempoSurg simulator was derived from high resolution CT-data of actual human temporal bones. Key point of this model is the high resolution of the haptic and the visual rendering. The model can be deformed using different cutting tools. Shutter glasses give the impression of a three-dimensional preparation. A corresponding navigation tool allows controlling the drilling process within the 3 planes.

Results: Many of the most demanding challenges of a virtual surgical simulator (position of surgeon, choice of temporal bones, opportunity to work with patient cases, degrees-of-freedom, and working with alternative instruments) have already been accomplished.

Conclusions: The VOXEL-MAN TempoSurg simulator allows unlimited, standardized and time- as well as cost-saving training for OR-readiness in temporal bone surgery. The training effect can be augmented using the DICOM CT-files of actual patient cases.

Key words: virtual reality surgical simulators, haptic and visual rendering, temporal bone drilling

[Accepted Jan. 11, 2008]

\section{INTRODUCTION}

There is a growing demand for the simulation of surgical interventions in medical training, in surgical planning as well as in the assessment of surgical qualification. Today it is possible to overcome this demand due to the progress of Virtual Reality (VR) techniques as well as to the availability of detailed three-dimensional anatomical models ${ }^{1)}$.

Gold standard of surgical education in general is the cadaver model. The traditional temporal bone

Correspondence: Rudolf Leuwer, M.D.,

Professor of Otorhinolaryngology, University of Düsseldorf Medical School at Krefeld, Head of ENT-Dept., Helios Hospital Krefeld, Lutherplatz 40, 47805 Krefeld, Germany

e-mail: leuwer@klinikum-krefeld.de 
(TB) laboratory is an excellent example of such a high fidelity, cadaver simulator ${ }^{2)}$. Although the laboratory is an indispensable part of otology training, there are growing ethical as well as legal restrictions for the access to cadaver specimen. In addition the preparation properties of the cadaver specimen depend on the fixation method. For most of the teaching programs, however, fresh specimens are inaccessible and mostly restricted to scientific purposes. That is why especially for Otorhinolaryngology it is necessary to pursue the development of a virtual reality surgical simulator.

The simulation of surgery of the skull base and midface has very special requirements: Due to the complexity of the surgical anatomy the anatomical model has to be of high accuracy, the visualization of the cutting process has to be very precise and - finally - the training effect should be enhanced by a haptic force-feedback ${ }^{3,4)}$.

\section{MATERIAL AND METHODS}

The VOXEL-MAN TempoSurg simulator (Spiggle and Theis Company, Overath, Germany; Nagashima Medical Instruments Co. Ltd., Tokyo, Japan) was developed at the University Medical Center Hamburg-Eppendorf, Hamburg, Germany. The anatomical 3D-models were derived from high resolution CT-data of human temporal bones (app. 156 slices, $512^{2}$ pixels, $1 \mathrm{~mm}$ slice thickness, $0.33 \mathrm{~mm} \times 0.33 \mathrm{~mm}$ pixel size). Within each of these models app. 30 objects (e.g. facial nerve, auditory ossicles, and labyrinth) were defined using a semi-automatic threshold-based segmentation approach. The VOXEL-MAN software allows to interactively cut into these particular models giving new cut surfaces with high anatomic accuracy. In addition, a 6 degrees of freedom haptic feedback device (Phantom Omni, SensAble Technologies, Inc., Woburn, MA, USA) was integrated into the system giving a haptic rendering of the anatomical model with the same resolution as the visual rendering. The operator uses a foot pedal to control the virtual "drill" and wears specialized shutter glasses to view the computer generated image in three dimensions. The "drill" changes the appearance of the $3 \mathrm{D}$-model in real time as if the temporal bone is being drilled. Using the haptic feedback device the operator experiences the change in pressure related to the material being cut. The degree of hardness of the preparation can be adjusted as well. The operator can select between 5 equally segmented temporal bones including temporal bone pathology (cholesteatoma, eburnated temporal bone). He can also import sequences of high resolution DICOM-CT images and create his own 3D-model of actual cases. The preparation process is accompanied by a navigation tool which allows controlling the drilling process within the 3 cross-sectional planes. Depending on the experience of the trainee, this navigation tool can be switched off ("examination mode"). Thus the simulator allows a step by step inductive learning of the temporal bone surgical anatomy as well as of the surgical dexterity.

\section{RESULTS}

In order to pursue the development of a virtual reality surgical simulator many tasks had to be translated into effect. Figure 1 gives an impression of a traditional TB laboratory: The choice of instruments is nearly the same as in an original OR-setting. The trainee has to dress like in an OR because of the biohazard and the laboratory has to be separate from the patients area. Figure 2 shows the simulator in a demonstration setting. Several people can watch the scene. The position of the surgeon is nearly the same as in the traditional laboratory. On the start screen (Figure 3) the trainee can choose between 5 fully segmented cases, and even import his own DICOM CT-files (patient cases). On Figure 4 the mastoid has been fully drilled ("eburnated case"). With the left 


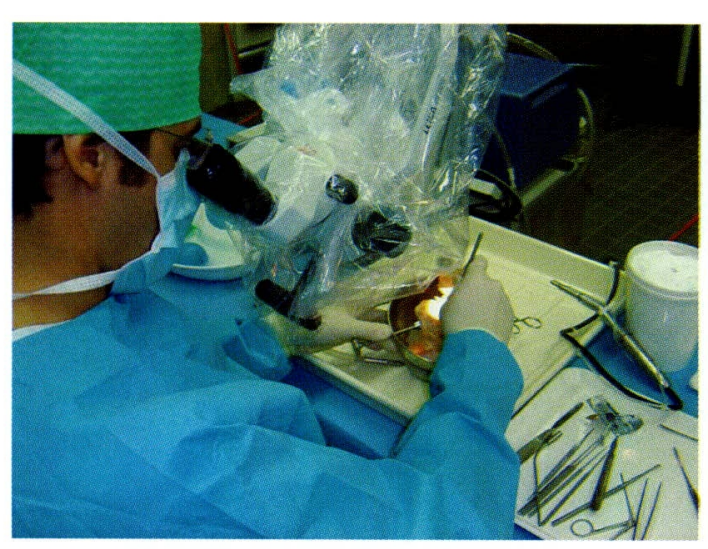

Fig. 1 Traditional temporal bone laboratory

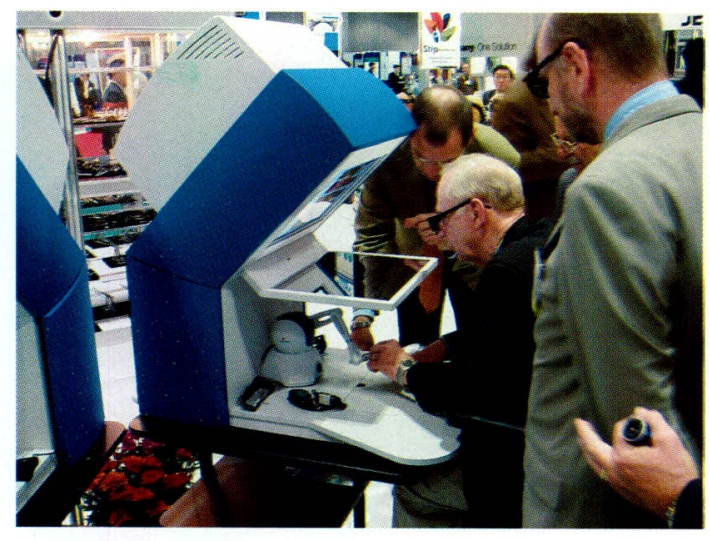

Fig. 2 Demonstration of the preparation on the VOXEL-MAN TempoSurg to several colleagues at the same time

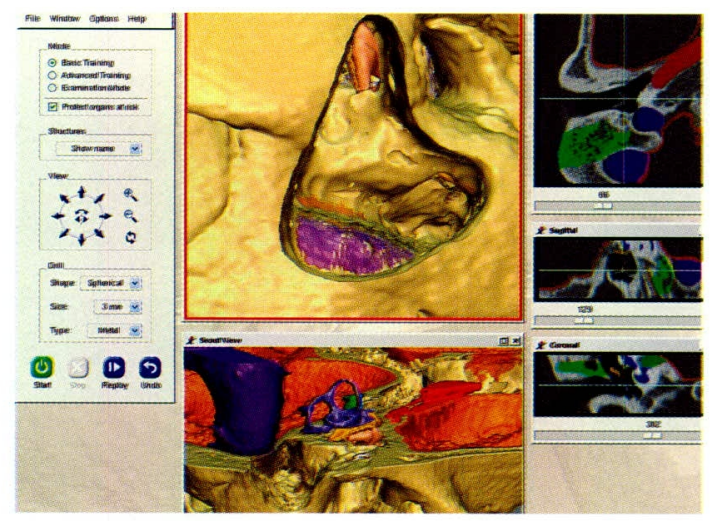

Fig. 4 Completed mastoidectomy on the "eburnated" case

panel the trainee can choose between different degrees of difficulty, he can turn and magnify the scene and he can take different kinds and almost unlimited sizes of drills. On the top window in the middle panel he can work out the temporal bone. The lower window gives him the same scene in a second plane. On the right navigation panel the trainee can control the bone that has already been removed (shown in green). Figure 5 shows a detailed view of a posterior tympanotomy. The cochleostomy has been completed with a $1 \mathrm{~mm}$ drill. The top window on the right panel demonstrates the exact position of the cochleostomy on the promontory. On Figure 6 a pars-tensa-cholesteatoma has been prepared. See the dehiscence of the facial nerve underneath the cholesteatoma.

For imported patient data, a set of CT acquisition parameters could be determined for which a very high resolution of the resulting 3D-models is obtained (Figure 7) ${ }^{5}$. The appearance of the bone can be optimized using a threshold on an additional control panel. As in the other cases, the model may be visually inspected and drilled on $3 \mathrm{D}$ views, and controlled on additional cross-sectional images.

For the development of other simulation topics it is possible to create varying virtual instruments (Figure 8). 


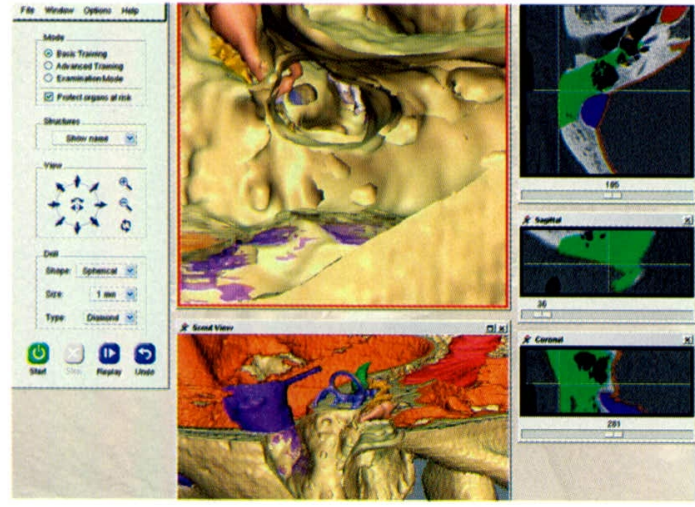

Fig. 5 Posterior tympanotomy with cochleostomy ("normal case")

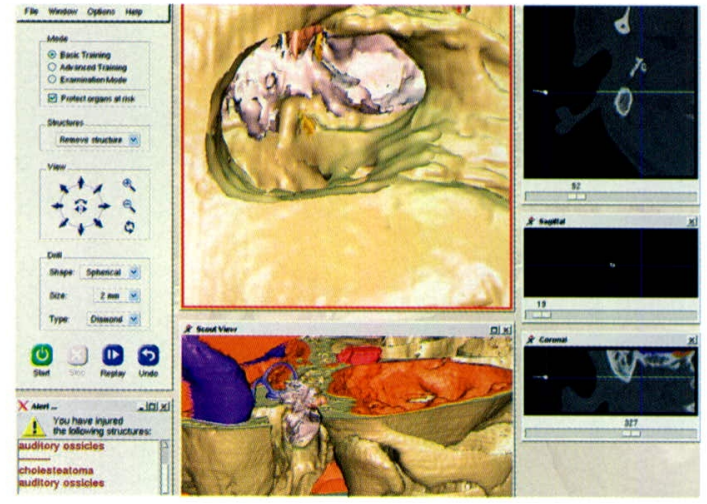

Fig. 6 Full preparation of the cholesteatoma

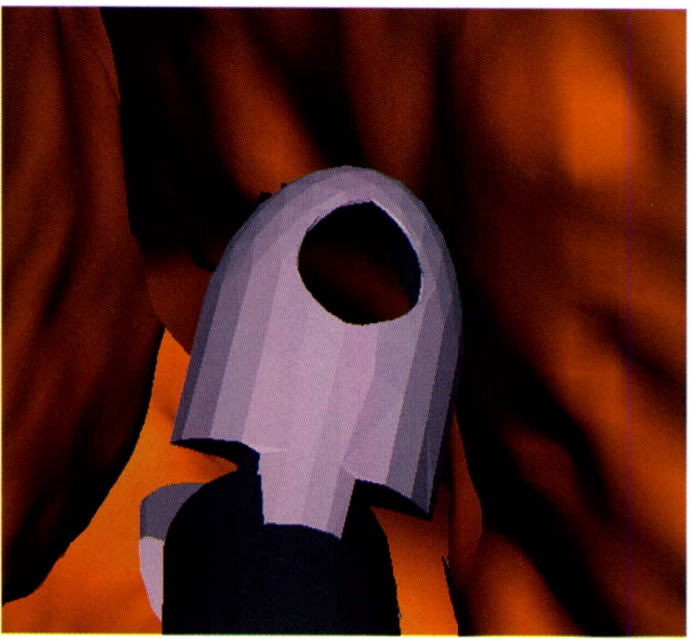

Fig. 8 Virtual surgery with a Blakesly forceps

\section{DISCUSSION}

In general, traditional cadaveric and virtual simulator temporal bone drilling provides a task that is fundamentally similar. To inductively work with a virtual model, however, is distinct from the traditional cadaveric temporal bone lab. With the simulator the task is standardized, and each parameter can be controlled (different types of pneumatisation, temporal bone pathology, different modes), allowing one to develop assessments that are not constrained by anatomic variation. Using the VOXELMAN TempoSurg model the trainee has the opportunity to make and to immediately correct mistakes.

In a recent study Zirkle et $\mathrm{al}^{6)}$. could show that the VOXEL-MAN TempoSurg simulator is appropriate for the assessment of trainees at the transition from laboratory based learning to operative practice. The authors inferred that having an objective assessment at this juncture might be useful for training programs, particularly if staff education time is limited. Although a temporal bone simulator is no substitute for operative experience with the complex preoperative decision making and planning, patient and microscope positioning, blood in the field etc., the authors expect that practice 


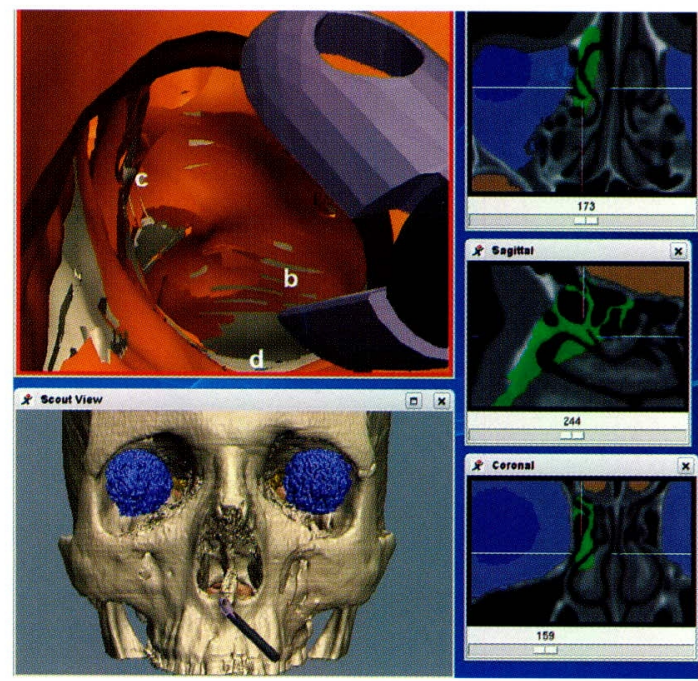

Fig. 9 Preparation screen of the new VOXELMAN SinuSurg simulator module

on a virtual reality temporal bone simulator will transfer to the operative setting in a similar fashion that traditional temporal bone laboratory does.

\section{PROSPECTS}

Very soon the VOXEL-MAN Group is going to introduce an additional simulator on the same platform: The SinuSurg simulator is another challenge for a systematical training in Otorhinolaryngology (Figure 9).

\section{REFERENCES}

1) Pommert A, Höhne KH, Burmester E, et al: Computer-based anatomy: A prerequisite for computer-assisted radiology and surgery. Acad Radiol $13: 104-112,2006$.

2) Zirkle M, Taplin MA, Anthony R, et al: Objective assessment of temporal bone drilling skills. Ann. Otol. Rhinol. Laryngol $116: 793-798,2007$.

3) Pflesser B, Petersik A, Tiede U, et al: Volume cutting for virtual petrous bone surgery. Comput Aided Surg; $7: 74-83,2002$.

4) Petersik A, Pflesser B, Tiede U, et al: Realistic haptic interaction in volume sculpting for surgery simulation. In: Ayache N, Delingette H (Eds.). Surgery simulation and soft tissue modelling. Proc. IS4TM 2003, Lecture notes in computer science 2673. Springer Berlin 194-202, 2003.

5) Tolsdorff B, Petersik A, Pflesser B, et al: Preoperative simulation of bone drilling in temporal bone surgery. Int J. CARS 2 (Suppl.) 160-161, 2007.

6) Zirkle M, Roberson DW, Leuwer R, et al: Using a virtual reality temporal bone simulator to assess otolaryngology trainees. Laryngoscope $117: 258-263,2007$. 